DR. INGE LEUNISSEN (Orcid ID : 0000-0002-3977-3620)

Article type : Research Report

\title{
Reliable estimation of inhibitory efficiency: To anticipate, choose, or simply react?
}

Inge Leunissen ${ }^{1}$, Bram B. Zandbelt², Zrinka Potocanac ${ }^{3}$, Stephan P. Swinnen ${ }^{1}$, James P. Coxon ${ }^{4}$

${ }^{1} \mathrm{KU}$ Leuven, Movement Control and Neuroplasticity Research Group, Belgium

${ }^{2}$ Centre for Cognitive Neuroimaging, Donders Institute for Brain, Cognition and Behaviour, Nijmegen, The Netherlands

${ }^{3}$ Jožef Stefan Institute, Department of Automation, Robotics and biocybernetics, Ljubljana, Slovenia

${ }^{4}$ School of Psychological Sciences and Monash Institute of Cognitive and Clinical Neurosciences, Monash University, Australia

\section{Corresponding author:}

Inge Leunissen

Tervuursevest 101

3001 Heverlee, Belgium

Tel.: +32 16 376440, Fax.: +3216329197

E-mail: inge.leunissen@kuleuven.be

Running title: Reliable estimation of inhibitory efficiency

Key words: response inhibition, stop-signal paradigm, response strategy

This article has been accepted for publication and undergone full peer review but has not been through the copyediting, typesetting, pagination and proofreading process, which may lead to differences between this version and the Version of Record. Please cite this article as doi: $10.1111 /$ ejn. 13590

This article is protected by copyright. All rights reserved. 


\section{Abstract}

Response inhibition is an important executive process studied by clinical and experimental psychologists, neurophysiologists, and cognitive neuroscientists alike. Stop-signal paradigms are popular because they are grounded in a theory that provides methods to estimate the latency of an unobservable process: the stop-signal reaction time (SSRT). Critically, SSRT estimates can be biased by skew of the response time distribution and gradual slowing over the course of the experiment. Here, we present a series of experiments that directly compare three common stop-signal paradigms that differ in the distribution of response times. The results show that the widely used choice response (CR) and simple response (SR) time versions of the stop-signal paradigm are particularly susceptible to skew of the response time distribution and response slowing, and that using the anticipated response (AR) paradigm based on the Slater-Hammel task offers a viable alternative to obtain more reliable SSRT estimates.

\section{Introduction}

The ability to rapidly prevent already initiated actions is essential in daily life. Many cognitive paradigms are used to index the construct of behavioral inhibition, but stop-signal paradigms are particularly popular because they come with a model that allows for estimation of the speed of the inhibitory process, i.e. the stop-signal reaction time (SSRT). This estimation is based on a race model in which stopping performance is dictated by the outcome of a race between two independent processes: a go process initiating the response, and a stop process inhibiting the response (Logan et al., 1984). The independent race model enables estimation of the finishing time of the stop process from the distribution of the go signal reaction times and the probability of responding on stop trials provided that two independence assumptions are met: 1) the go process is equivalent on both go trials and stop trials, i.e., it is not affected by the presentation of the stop-signal (context independence), 2) trial-by-trial variability in go RT is unrelated to trial-by-trial variability in SSRT (stochastic independence). In practice, researchers 
use either a set of fixed stop-signal delays (SSD) or a staircasing procedure, the latter having the intention to reach an average probability of responding to a stop signal of 0.5 . The two most common ways to calculate SSRT are the mean method and the integration method. In the mean method the average delay between the go signal and stop-signal (i.e. stop-signal delay, SSD) is subtracted from the mean go response time. In the integration method the go response time distribution is integrated to find the point where the integral equals the probability of responding, SSRT is then calculated by subtracting the mean SSD from this nth response time (Verbruggen \& Logan, 2009). Logan et al. (1997) have shown that using a tracking algorithm and the mean method results in more reliable SSRT estimates than applying the integration method to estimate SSRT per fixed SSD. Simulations and reliability tests suggest that both mean and integration estimates of SSRT are reliable for the central SSDs (where P(stop success) is close to 0.5) (Band et al., 2003; Congdon et al., 2012). However, Verbruggen et al. (2013) showed that skewness of the response time distribution and response slowing in anticipation of stop-signals can still lead to biased SSRT estimates, especially under the mean method.

In the past 5 years, $82.5 \%$ of published studies reporting a stop-signal paradigm used a choice response (CR) as the primary "go" task and 10.4\% used a simple response (SR) (see Appendix I for search details). In these reaction time versions, participants respond to a go signal and attempt to prevent the response when a stop-signal is infrequently presented after the go signal (Lappin \& Eriksen, 1966; Logan et al., 1984)(Figure 1). The other 7.1\% made use of an anticipated response (AR) paradigm. In the AR paradigm the stop-signal is presented before an anticipated response (Slater-Hammel, 1960; Coxon et al., 2006). The primary "go" task is to respond when a moving indicator reaches a stationary target. The requirement for behavioral inhibition is signaled by automatically stopping the indicator prior to the target.

The CR, SR and AR stop-signal paradigms are similar in that the participant is asked to accomplish two competing goals in both tasks: respond to the go stimulus and withhold this response if a stop-signal is presented. However, the CR/SR and AR versions of the stop-signal task differ in an important way. The CR/SR stop-signal paradigms involve speeded responses,

This article is protected by copyright. All rights reserved. 
whereas the AR paradigm involves timed responses. Because the response in the AR task has to occur at a known point in time the paradigm ensures relatively consistent initiation of the go response in the presence of stop-signals. It also provides the participant with direct feedback about how close they stopped the indicator relative to the target on each trial, reinforcing correct go task performance. In contrast, in the CR/SR paradigm there is no hard time constraint to the response process, other than the experimenter's instruction to respond as fast and accurately as possible (although some studies impose deadlines, e.g., Mirabella et al. (2006), or rewards, e.g., Ridderinkhof et al. (1999)). Consequently, there may be more room for response strategy adjustments aimed at increasing the chance of success on stop trials in the CR/SR paradigm than in the AR paradigm.

The presence of a strategic bias as a potential nuisance factor has been acknowledged since the CR/SR paradigm's inception (Logan et al., 1984). Emphasis on going fast results in longer SSRTs, whereas focusing on stopping correctly can shorten SSRTs (Leotti \& Wager, 2010; Greenhouse \& Wessel, 2013). This might sound sensible, however in order to increase the chance of stopping participants tend to slow down their go responses, resulting in a more skewed response distribution and often a gradual increase in go response times over the course of the experiment, both factors that can bias the SSRT estimation (Verbruggen et al., 2013).

Hence, individual differences in the trade-off to go fast or to stop accurately may hamper the straightforward interpretation of the SSRT, which made the use of the stop-signal task so popular. This is particularly relevant when comparing groups with systematic differences in speed-accuracy trade-offs, such as the elderly, who are known to adopt a more cautious response strategy than young adults (Ratcliff et al., 2007; Forstmann et al., 2011).

In Experiment 1 we directly compared, in the same participants, the most widely used CR task with the AR task. We evaluated response time distributions using ex-Gaussian distribution analysis, response slowing over time, and critically, the reliability of the SSRT estimation. In addition, we tested whether the AR paradigm meets the assumptions of the race model.

This article is protected by copyright. All rights reserved. 
Besides the difference in time constraint on the response process between the CR and AR tasks, the CR paradigm also measures choice reaction times, whereas the AR task measures simple response times. Logan et al. (1984) showed that the latency of the stop process increases when the go task involves a choice reaction as opposed to a simple response task. To address if any differences between the CR and AR task found in Experiment 1 could be explained by the decision component in the CR task, we compared the AR task with the SR version of the stopsignal paradigm in Experiment 2. In this experiment, we also included a group of older participants to investigate the influence of response strategy (the age-related tendency to prioritize accuracy over speed). Despite the differences in the primary go task, we expected a positive correlation for stopping performance (SSRT) across tasks, which would be consistent with a common underlying neural mechanism.

\section{Methods}

\section{Experiment 1}

\section{Participants}

Eighteen participants (age: mean 23.5yr, range 19-30; 6 male) without neurological impairments were recruited for this experiment. The University Hospital Leuven ethics committee approved this study, and written informed consent was obtained prior to the experiment.

\section{Procedure}

Participants were seated $1 \mathrm{~m}$ in front of a 19 -in computer screen with at $60 \mathrm{~Hz}$ refresh rate, positioned at eye level. For the AR stop-signal task, the right hand was tested. For the CR stop-signal task, participants made a choice reaction with their left or right hand. In our previous studies with the AR task participants were instructed to release a button (Coxon et al., 2007; 2009; 2012; Leunissen et al., 2016) or press a button (Zandbelt \& Vink, 2010; Zandbelt et

This article is protected by copyright. All rights reserved. 
al., 2011; 2013a; 2013b) when a moving indicator reached a stationary target. In the CR task participants typically have to press the button corresponding with the correct decision. To test whether pressing or releasing the button during the go task has a meaningful influence on stop task performance, we utilized a $2 \times 2$ within-subjects factorial design, with levels task (AR, CR) and response (press, release).

\section{Anticipated response (AR) version of the stop-signal task}

The visual display consisted of a vertical indicator that moved from the bottom upwards at an equal and constant rate on each trial. A horizontal target line was situated $800 \mathrm{~ms}$ from onset (Figure 1a). The primary task was to try to stop the indicator at the target line (go trials) as accurately and consistently as possible. To reinforce go task performance the color of the target line changed to green, yellow, orange or red at the end of the trial, depending on whether responses were within $20,40,60$, or $>60 \mathrm{~ms}$ of the target. In $30 \%$ of the trials the indicator was programmed to stop automatically prior to reaching the target. When this happened, participants tried to prevent their response (stop trials). A staircasing algorithm ensured convergence to $50 \%$ success on stop trials. The initial stop time was set to $180 \mathrm{~ms}$ from the target line (i.e. 800-180ms) based on previous studies (Coxon et al., 2006) and stop time was adjusted in steps of $16 \mathrm{~ms}$, which was half the average go response time standard deviation, based on previous studies (Coxon et al., 2006) and pilot data.

\section{Choice reaction (CR) version of the stop-signal task}

As many features as possible were kept the same between the AR and CR stop-signal tasks, e.g., trial timing and order, and the number of stop trials. An arrow was presented on the screen, which pointed towards the left or towards the right (Figure 1b). The primary task was to press/release the switch corresponding with the direction of the arrow (go trials). Participants were instructed to respond as fast and as accurately as possible. In $30 \%$ of the trials a square would appear shortly after, replacing the arrow. When this happened, the participant tried to prevent their response (stop trials). Two separate staircasing algorithms were used for the left

This article is protected by copyright. All rights reserved. 
and right hand to ensure equal numbers of successful and failed stop trials for each hand. The initial stop-signal delay was set to $180 \mathrm{~ms}$ and was adjusted in steps of $35 \mathrm{~ms}$. Again, stop time adjustment was set at half the average go response time standard deviation, based on pilot data.

\section{Common task instructions}

For both tasks, participants were instructed to rest their index finger(s) on modified computer mouse switch(es) (press condition) or to depress the switch(es) using only as much force as was necessary to keep the switch depressed (release condition). For the press condition of both tasks the forearms were pronated with the index fingertips positioned on the computer mouse keys (Fig. $1 \mathrm{c}+\mathrm{d}$ ). In the release condition, the forearms were semiprone with the medial aspect of the index fingertips positioned on the computer mouse keys (Figure $1 \mathrm{c}+\mathrm{d}$ ).

It was emphasized that go trials were to be performed as accurately as possible (a speed instruction was also given for the CR task, as described above). Regarding stop trials, participants were told 1) to try to prevent their response whenever they could, 2) that sometimes they would not be able to prevent pressing/releasing the switch, and not to worry when this happened as the experiment was designed to determine how much time they needed to prevent movement.

For each of the four task/response combinations, participants first practiced go trials, then go + stop trials $(\sim 5 \mathrm{~min})$. Next they performed a block consisting of only go trials (50 trials, $\sim 5 \mathrm{~min}$ ), followed by three task blocks of that condition, each consisting of 100 trials (in total 210 go and 90 stop trials, $\sim 20 \mathrm{~min}$ ). The inter-trial interval (ITI) randomly varied between 3 and

4s. The task/response configuration order was pseudo-randomized partial counterbalanced and split across two collection days (on average 5.6 days apart, range 1-22). Responses were recorded with custom LabView software, which also generated the visual display.

This article is protected by copyright. All rights reserved. 


\section{Dependent measures}

For each participant, mean go trial response times (GoRT), the standard deviation of go response times and response times for failed stop trials were determined. For the AR task response times are reported relative to the target (time of response $-800 \mathrm{~ms}$, e.g. if response occurred at $824 \mathrm{~ms}$, then $824-800=24 \mathrm{~ms}$ after the target), whereas for the CR task they are reported relative to imperative cue onset. Early response times (AR $<400 \mathrm{~ms}$, CR $<150 \mathrm{~ms}$ ), and trials with an incorrect response were considered errors. Go trials on which no response was made were excluded. Response times more than 3 standard deviations from mean GoRT were defined as outliers.

The independent race model predicts that only the fastest go processes are able to escape inhibition, therefore response times of failed stop-signal trials should be shorter than response times of go trials. To visually depict this we plotted the cumulative distributions of response times for both go trials and failed stop trials. In addition, response times of failed stop trials should increase as a function of stop-signal delay due to the stop process finishing later. We tested this by means of a linear regression and illustrated it by plotting the mean stop fail RT as a function of stop-signal delay. We performed a linear mixed effects analysis of the relationship between mean stop fail RT and stop-signal delay. As fixed effects we entered stopsignal delay, task and response mode into the model, and random intercepts were modeled at subject level (R package nlme version 3.1-128 (Pinheiro et al., 2013)).

To assess slowing over time we used a similar linear mixed effects analysis with fixed effects time, task and response mode and random intercepts at subject level. DISTRIB v2.3 (Lacouture \& Cousineau, 2008) was used to provide ex-Gaussian estimates. The ex-Gaussian distribution can be used to more accurately describe RT data (Ratcliff \& Murdock, 1976). It results from the convolution of a Gaussian function and an exponential distribution and is characterized by three parameters: mu (mean of the Gaussian component), sigma (standard deviation of Gaussian component), and tau (both the mean and standard deviation of the exponential component). Tau $(\tau)$ was the parameter of interest since it reflects the length of the

This article is protected by copyright. All rights reserved. 
right-hand tail of the RT distribution. In addition we report the ratio between tau and sigma to quantify skewness after standardization of scale (Heathcote et al., 2002). To visualize the skewness of the GoRT distribution we plotted the individual, and group ex- Gaussian probability density functions. The group probability density function was obtained by averaging the individual parameter estimations across the group.

For stop trials, the probability of responding (p(respond)|stop) was calculated. The stop-signal reaction time (SSRT) was determined via the integration method (Verbruggen \& Logan,

2009). The mean stop-signal delay was subtracted from the GoRT at the nth percentile of the distribution of correct go trial RTs, where $\mathrm{n}$ is the participant's probability of responding (p(respond)|stop).

\section{Statistical analysis}

Dependent measures were subjected to analysis of variance (ANOVA) for repeated measures with factors task (AR, CR) and response (press, release) in IBM SPSS Statistics version 21 (IBM Corp., Armonk, N.Y., USA). It was verified that each participant had a probability of responding on stop trials between $40-60 \%$ and that mean RT on failed stop trials was shorter than mean RT on go trials. One participant was identified as a significant outlier based on SSRT values (Grubb's test $\mathrm{p}<0.05$ ) and was removed from statistical analysis to satisfy the assumption of normality for inferential parametric statistics. The criterion for statistical significance was $\alpha=0.05$. Post hoc Bonferroni-corrected paired t-tests were used to test interactions.

To address the question whether inhibitory performance on the AR task relates to inhibitory performance on the CR task, one-tailed correlations between SSRTs were performed. Reported p-values are Bonferroni corrected for the number of correlations performed per dependent measure.

Finally, the internal reliability of the SSRT estimation was analyzed. Go and Stop trials

This article is protected by copyright. All rights reserved. 
were randomized and split in half. This procedure was repeated 500 times per subject, for Go and Stop trials separately, to avoid any one randomization driving the results. The absolute mean difference between SSRT values estimated from all random halves of the data and the within-subject standard deviation were calculated:

$$
\sigma_{w}=\sqrt{\frac{\sum_{i=1}^{n} \sigma_{i}^{2}}{n}}
$$

where $\sigma_{i}^{2}$ is the variance of the difference in SSRT estimation between random halves of the data for subject $i$, and $n$ denotes the total number of participants in the sample. Based on $\sigma_{w}$, the coefficient of repeatability of the SSRT calculation was estimated, i.e. the difference between two SSRT estimations for the same subject is expected to be less than $\sqrt{2} * 1.96 \sigma_{w}$ for $95 \%$ of the pairs of random halves of the data (Bland \& Altman, 1996).

\section{Experiment 2}

\section{Participants}

Fourteen young adults (age: mean 24.5 yr, range 21-33; 6 male) and twelve elderly participants (age: mean $71.9 \mathrm{yr}$, range 66-78; 7 male) without neurological impairments were recruited for this experiment. The University Hospital Leuven ethics committee approved the study, and written informed consent was obtained prior to the experiment.

\section{Procedure and statistical analysis}

Participants performed an anticipated response (AR) and a simple reaction (SR) version of the stop-signal task. Both tasks were performed on the same day in a counterbalanced order. The basic trial structure, instructions and analyses were identical to those from Experiment 1 with the following exceptions: In the AR task the color of the target line was white and did not change according to go task performance (i.e. no reinforcing feedback was given) and participants always responded by releasing the mouse switch. In the SR task the go stimulus 
was a green square, which required pressing the button as fast as possible. In $30 \%$ of the trials the green square change into a red square, signaling that participants should try to prevent their response (Figure 1c). The initial stop-signal delay for both task paradigms was set to $200 \mathrm{~ms}$. The staircasing algorithm was adjusted in steps of $25 \mathrm{~ms}$ for the AR task and $50 \mathrm{~ms}$ for the SR task. No blocks containing just go trials were collected (i.e. there was no "go only" block). Dependent measures were subjected to analysis of variance (ANOVA) for repeated measures with factors task (AR, SR) and group (young, old). One young participant was removed from all statistical analysis due to a probability of responding on stop trials of only $15 \%$ in the SR task.

\section{Results}

\section{Experiment 1}

Table 1 shows performance and associated statistics of the AR and CR versions of the stopsignal paradigm for both the press and release conditions.

\section{Independence of the go and stop process}

The effect of trial type on response time was assessed using ANOVA with trial type (go trial, failed stop trial), task (AR, CR) and response mode (press, release) as within-subject variables. Response times on failed stop trials were significantly faster than response times on go trials in both tasks $(F(1,16)=121.03, p<0.001, \eta 2=0.88)$, but in absolute values the difference between response times on failed stop trails and go trials was larger in the CR task (trial type by task interaction: $F(1,16)=40.77, p<0.001, \eta 2=0.72)$. The main effect of press/release condition did not reach statistical significance $(F(1,16)=0.39, p=0.541, \eta 2=0.02)$, and neither did the other interaction effects (trial type by response mode $(F(1,16)=1.03, p=0.325, \eta 2=0.06)$, task by response mode $(F(1,16)=0.55, p=0.47, \eta 2=0.03)$, trial type by task by response mode $(F(1,16)=3.70, p=0.07, \eta 2=0.19))$. Cumulative RT distributions are presented in Figure 2a.

Response times on failed stop trials (SF) increased with the increase in stop-signal delay (SSD) for both tasks (AR press: $\beta=0.52$; AR release: $\beta=0.58$, CR press: $\beta=0.45$, CR release: $\beta=0.36$, 
effect of task: $F(1,16)=1.36, p=0.24$, effect of response mode: $F(1,16)=4.33, p=0.04$, task by response mode interaction: $F(1,16)=0.00, p=0.98$, Figure $2 b)$. Together, these results support context independence (Logan et al., 1984; Verbruggen \& Logan, 2009) between the go and stop process in both the AR and CR task, meaning that independent race model will provide valid estimates of SSRT for this data set.

\section{Go performance}

Errors, such as responding too early (intentionally or accidentally), were rare but occurred more frequently in the release than press condition in both tasks (Table 1). The percentage of Go trials in which no response was made was $<1 \%$ in all task conditions. GoRT variability was higher in the CR version of the stop-signal paradigm. This is further exemplified by the significant interaction between the factors condition (go only, go in the presence of a potential stop-signal) and task $(A R, C R)(F(1,16)=23.66, p<0.001, \eta 2=0.60)$. Post-hoc testing revealed that GoRT variability substantially increased when introducing stops in the CR task $(\mathrm{p}<0.001)$, whereas no such effect on GoRT variability was detected in the AR task $(p=0.971)$.

We found a significant difference in GoRT slowing over the course of the experiment between tasks (Table 1). On average, slowing over time did not seem to be a factor in either task (average coefficients, AR: $\beta=-0.015 \mathrm{~ms} /$ trial, $\mathrm{CR}: \beta=0.07 \mathrm{~ms} /$ trial). However, the range of $\beta$ (AR: [-0.16 to 0.05$]$, CR: [-0.42 to 0.84$]$ ) indicates that slowing over time is minimal for the AR task, whereas in the CR task the values go up to $0.84 \mathrm{~ms} /$ trial, reflecting a $250 \mathrm{~ms}$ increase in GoRT over the course of 300 trials (Figure 3a upper panel).

To quantify the skewness of the GoRT distributions on an individual level, we estimated tau, and the ratio between tau and sigma, and performed a 2x2x2 ANOVA with the factors condition (go only, go in the presence of a potential stop-signal), task (AR, CR) and response mode (press, release) (Figure 3a lower panel). There was no significant condition by task interaction for either measure of skewness (tau: $F(1,16)=0.107, p=0.748, \eta 2=0.01$, tau/sigma: $F(1,16)=3.251, p=0.090, \eta 2=0.169)$, indicating that tau increased with the presence of stop 
signals in a similar way for both tasks. There was a significant main effect of the presence of stop-signals for tau $(F(1,16)=7.908, p=0.013, \eta 2=0.33)$, but not for tau/sigma $(F(1,16)=0.369$, $p=0.552, \eta 2=0.03)$. In contrast, there was a significant task effect between the AR and CR stopsignal paradigms for both measures of skewness (tau: $F(1,16)=140.822, p<0.001, \eta 2=0.90$, tau/sigma: $F(1,16)=43.144, p<0.001, \eta 2=0.73)$. Individual and group ex-Gaussian probability density functions are shown in Figure $3 b$.

\section{Stop performance}

The dynamic tracking procedure was successful in obtaining P(stop success) close to $50 \%$ in both paradigms. The mean percentage of successful stop trials was $52.5 \%$ for the CR version and $50.7 \%$ for the AR task, a subtle but statistically significant difference (Table 1 ). SSRTs were significantly longer in the CR task than in the AR task. Moreover, participants required more time to inhibit their response in the release than press condition. The task by response interaction was not significant for SSRT values (Table 1).

To exclude the possibility that the SSRT measures were biased due to any temporal order effects we performed a 4x2x2 ANOVA with factors data collection order, task (AR, CR) and response mode (press, release) and SSRT as dependent measure. We found no evidence for such an effect, as the main effect of order $(F(1,16)=0.018, p=0.894, \eta 2=0.00)$, the order by task interaction $(F(1,16)=0.262, p=0.610, \eta 2=0.01)$, the order by response mode interaction $(F(1,16)=0.030, p=0.863, \eta 2=0.00)$, and the three-way interaction $(F(1,16)=0.003, p=0.0 .957$, $\eta 2=0.00$ ) were not significant.

To test for the relationship between SSRT measures of the different task conditions we performed three correlation analyses, (1) between the AR and CR stop-signal tasks, collapsed over press and release conditions, (2) between press and release condition of CR task, (3) between press and release condition of AR task. We found a significant positive relationship between the SSRTs of the AR and CR task ( $r=0.48, p=0.025)$ (Figure 4a). A person who was fast and efficient at stopping in the AR task was also fast and efficient at stopping in the CR task.

This article is protected by copyright. All rights reserved. 
When comparing SSRT measures for press and release conditions within-task, a significant correlation was observed for the CR task ( $\mathrm{r}=0.58, \mathrm{p}=0.008$ )(Figure $4 \mathrm{~b}$ ) but not the AR task $(r=0.23, p=0.192)$ (Figure 4c). To explore this further, we used Zou's (Zou, 2007) method to calculate the $95 \%$ confidence interval of the difference between the correlations. The confidence interval for rCR - rAR was [-0.25, -0.92], indicating a significant difference between the correlation coefficients. However, an additional computer simulation (Appendix II) suggests that the low correlation between AR press and AR release may be a reflection of low betweensubjects variability (restricted range) and does not discount the presence of a "true" correlation in the population.

\section{SSRT reliability}

The stability of SSRT estimates between repeated random splits was higher for the AR than the CR task (Table 1). The average absolute difference between the SSRT estimates of the pairs of random halves was more than twice as high in the CR than in the AR task. Across response modes, the within-subject standard deviation was $8.79 \mathrm{~ms}$ for the AR task and $24.99 \mathrm{~ms}$ for the CR task. The coefficient of repeatability was $24.33 \mathrm{~ms}$ in the AR task, i.e. for $95 \%$ of all random split halves pairs the difference in SSRT estimation was less than $24.33 \mathrm{~ms}$. For the CR task the coefficient of repeatability of SSRT was $69.23 \mathrm{~ms}$.

\section{Experiment 2}

Table 2 shows performance and associated statistics of the AR and SR versions of the stopsignal paradigm in younger and older adults.

\section{Independence of the go and stop process}

The effect of trial type on response time was assessed using 2x2x2 ANOVA with the factors trial type (go trial, failed stop trial), task (AR, SR) and group (young, old). Response times on failed stop trials were significantly faster than response times on go trials $(F(1,23)=241.302, p<0.001, \eta 2=0.91)$. Response times of older participants were slower than of 
the young adults $(F(1,23)=8.037, p=0.009, \eta 2=0.26)$. There was no trial type by group interaction $(F(1,23)=1.613, p=0.217, \eta 2=0.07)$, or a three-way interaction effect $(F(1,23)=2.108$, $\mathrm{p}=0.160, \eta 2=0.08)$. Cumulative RT distributions are presented in Figure $5 \mathrm{a}$.

Response times on failed stop trials increased with the increase in stop-signal delay for both tasks and groups (AR young: $\beta=0.63$, AR old: $\beta=0.82$, SR young: $\beta=0.52$, SR old: $\beta=0.58$, effect of task: $\mathrm{F}=0.00, \mathrm{p}=0.985$, effect of group: $\mathrm{F}=3.06, \mathrm{p}=0.08$, task by group interaction: $\mathrm{F}=0.26, \mathrm{p}=0.608$ )(Figure $5 \mathrm{~b}$ ). These results suggest context independence between the go and stop process in both the AR and SR task.

\section{Go performance}

Errors, such as responding too early (intentionally or accidentally), were rare but occurred more frequently in the AR than in the SR task, and older participants made more errors than young adults (Table 2). The percentage of Go trials in which no response was made was also rather high in older group (AR: 11\%, SR: 7\%). GoRT variability was higher in the SR version of the stop-signal paradigm, suggesting that also in the SR task go task performance is less well constrained than in the AR task. There was no significant main effect of group or a group*task interaction (Table 2).

There was significantly more GoRT slowing over the course of the experiment in the SR task compared to the AR task. There was no main effect of group or a task by group interaction (Table 2, Figure 6a upper panel).

To quantify the skewness of the GoRT distributions on an individual level, we estimated tau, and the ratio between tau and sigma, and performed a 2x2 ANOVA with the factors task (AR, SR) and group (young, old)(Figure 6a lower panel). The response distribution was significantly more skewed in the SR than in the AR task. There was no significant main effect of group or a task*group interaction (Table 2). Individual and group ex-Gaussian probability density functions are shown in Figure $6 \mathrm{~b}$.

This article is protected by copyright. All rights reserved. 


\section{Stop performance \& reliability}

The dynamic tracking procedure was successful in obtaining P(stop success) close to $50 \%$ in both paradigms. There were no significant differences in SSRT between tasks or groups (Table 2). However, SSRT estimates in the AR task and SR task correlated significantly in the young adults $(\mathrm{r}=0.69, \mathrm{p}=0.005)$ but not in the elderly $(\mathrm{r}=-0.12, \mathrm{p}=0.358)$ (Figure 7$)$, suggesting that one of the SSRT estimates might not be a reliable indicator of stopping ability. Indeed, the stability of SSRT estimates between repeated random splits was much better for the AR than the SR task (Table 2). Averaged across groups, the within-subject standard deviation was $14.72 \mathrm{~ms}$ for the AR task and $77.25 \mathrm{~ms}$ for the SR task. The coefficient of repeatability was $40.78 \mathrm{~ms}$ in the AR task, i.e. for $95 \%$ of all random split halves pairs the difference in SSRT estimation was less than $40.78 \mathrm{~ms}$. For the SR task the coefficient of repeatability of SSRT was $213.98 \mathrm{~ms}$.

The low repeatability of the SSRT estimate in the SR task could have consequences. If we had only collected the AR task in this sample and tested for a group difference in SSRT we would have concluded that there was a significant group difference with a large effect size $(t(23)=-$ 4.154, $\mathrm{p}<0.001, \mathrm{~d}=1.64)$, whereas for the SR task the group effect was not significant $(\mathrm{t}(23)=-$ 1.207, $\mathrm{p}=0.240, \mathrm{~d}=0.49)$.

\section{General discussion}

The CR and SR versions of the stop-signal paradigm are widely used in studies of response inhibition. They have been instrumental in bringing this important aspect of cognitive control to the attention of the scientific community and in formalizing measures to quantify inhibitory control. This study aimed to compare performance on the CR/SR to a less commonly used AR version of the stop-signal paradigm, and to validate that the AR task meets the requirements of the independent race model. The results show that the AR task indeed meets the requirements of the race model and is therefore an appropriate paradigm to test behavioral response inhibition. Moreover, the go response distribution in the AR task is less skewed and

This article is protected by copyright. All rights reserved. 
there is less gradual slowing over time than in the CR and SR paradigms, and the AR task provides more reliable SSRT estimates.

\section{The anticipated response task meets the requirements of the race model}

The independent horse-race model predicts that RTs on failed stop trials should be faster than RTs on go trials, because failed stop RTs only represent those responses that were fast enough to finish before the end of the stop process (Logan et al., 1984; Verbruggen \& Logan, 2009). In other words, the failed stop RTs should be similar to the fastest RTs in the GoRT distribution, indicating that the go process is equal during stop and go trials. The cumulative response distributions of all three paradigms indeed show that RTs on failed stop trials and GoRTs have a common minimum, but that GoRTs diverge to the right of the failed stop RT distribution due to the slower responses in the distribution (Figure 2a \& 5a). The independent horse-race model also predicts that RTs on failed stop trials should increase when the stopsignal delay increases. When the stop cue is presented close to the average go response onset, only the fastest go responses will escape inhibition, but when the stop cue is presented earlier, slower responses might also dodge inhibition. This pattern was confirmed in all three paradigms (Figure $2 \mathrm{~b} \& 5 \mathrm{~b}$ ). Note that data from simple stop-signal paradigms as used here are generally consistent with the independence assumptions of the horse-race model, but more complex stop-signal paradigms, such as selective stopping tasks, have been shown to violate the context and stochastic independence assumptions (Bissett \& Logan, 2014; Verbruggen \& Logan, 2015). Such violations should not be taken lightly, as they invalidate SSRT estimates. Therefore, future studies should explore whether more complex versions of the AR stop-signal paradigm still meet the independence assumptions.

This article is protected by copyright. All rights reserved. 


\section{Direct comparison of the AR and CR/SR tasks reveals meaningful differences in primary go task performance and SSRT reliability}

In Experiment 1 we compared performance on the most frequently used CR version of the stop-signal task and the AR version. The most obvious difference between the two paradigms is the primary go task. On the one hand, the go task is less constrained in the CR task as compared to the AR task. Consequently, the $\mathrm{CR}$ task leaves more room for response strategies, such as "waiting" for a possible stop-signal or preparing a more cautious response in an attempt to increase their success rate on the stop trials. In the AR task the go task is more tightly controlled because the response has to happen at a known point in time. On the other hand, the CR paradigm involves go-stimulus discrimination and thus measures choice reaction times, whereas the AR task involves detection, hence it measures timed responses.

For the same participants the introduction of stop cues had very different effects on go task performance. GoRT variability substantially increased when introducing stops in the CR paradigm, whereas GoRT variability demonstrated no significant difference in the AR task. In addition, the GoRTs in the CR paradigm were more positively skewed and some participants showed significant slowing over time. These differences are relevant as the simulations of Verbruggen et al. (2013) have demonstrated that skew and slowing over time affect the accuracy and reliability of the SSRT estimation. Bias due to skew of the response distribution may be circumvented by applying the integration method, however, in the presence of gradual slowing the integration method will still underestimate SSRT, even when only considering participants with a probability of inhibition close to 50\% (Verbruggen et al., 2013). Here, the dynamic tracking procedure was successful in obtaining a stop success rate close to $50 \%$ in both paradigms, but the within-subject standard deviation of the SSRT estimate was two times smaller in the AR than in the CR task, suggesting that the AR task provides more reliable SSRT estimates than the CR task.

Finally, SSRTs were longer for the release than the press condition in both the CR and AR paradigms. Similarly, the latency of the stop process increases when the go task involves a

This article is protected by copyright. All rights reserved. 
choice reaction as opposed to a simple response task (Logan et al., 1984; Szmalec et al., 2009). This suggests that task specific processes, such as response selection, error monitoring and response mode (press/release) can influence the latency of the stop process. The difference between the press and release respond modes is the moment at which the response is registered. When recording the release of a button one registers the beginning of a movement, whereas a press registers the end of a movement. Registering the beginning of the movement results in slightly more errors (such as responding too early or with the wrong effector, Table 1).

It is difficult to determine whether the differences in go task performance and SSRT reliability between the AR and CR task are attributable to strategic effects, or to the choice element present in the CR but not the AR task. Therefore we compared a simple reaction (SR) version of the stop-signal paradigm with the AR version in Experiment 2. The results show that, also in the SR task, go responses are more variable than in the AR task. The go response distribution is more skewed and there is appreciable slowing over time in about half of the participants $(>0.35 \mathrm{~ms} /$ trial, i.e. $>100 \mathrm{~ms}$ over the course of 300 trials, range SR: [-0.59 to 2.07], range AR: [-0.06 to 0.36]). The reliability of the SSRT measure was very poor in the SR task, in the young group the within-subject variability was eight times lower in the AR task than in the SR task and for the older participants three times. This leads us to conclude that the observed differences in go task performance and SSRT reliability between the AR and the CR task are likely due to the lack of a hard time constraint on response process in the CR task as opposed to the choice dimension.

The main problem with the SR task is that participants might not be able to stop without waiting for some period to see if stop-signal will appear because the typical reaction time in a simple RT experiment is around 200ms (Woodworth \& Schlossberg, 1954), which is in the same range, or even shorter than the typical SSRT (Logan et al., 1984). Note that the average response time in the SR task was $430 \mathrm{~ms}$, suggesting that participants indeed appear to have used a waiting strategy. This might drive participants to deliberate decision-making, i.e. on some trials

This article is protected by copyright. All rights reserved. 
they might decide to respond immediately, while on others they wait to see if a stop-signal will occur (Leotti \& Wager, 2010). To obtain a valid SSRT estimation, it is crucial that a go response is initiated, because in its absence there is no need to trigger the stop process. The AR task has been shown to meet this response initiation criterion, as the increase in motor cortex excitability is indistinguishable on go and stop trials prior to the occurrence of the stop cue (Coxon et al., 2006). Future studies could make use of recent approaches such as the Bayesian approach for estimating the probability of trigger failures (Matzke et al., 2016) or process models that give more insight into the mechanisms that implement going and stopping (Dunovan et al., 2015; Matzke et al., 2016).

Skew and slowing can be especially detrimental when comparing groups and conditions where strategic differences could be expected. For example, studies have shown that children with ADHD have longer SSRTs than children without ADHD (Oosterlaan et al., 1998). However, a recent study demonstrated that also tau is much higher in children with ADHD, producing spurious differences in SSRT due to biased estimates (Epstein et al., 2011). Here, we used a dataset that included groups of young adults and older adults with known differences in speedaccuracy trade-off (Ratcliff et al., 2007; Forstmann et al., 2011) to demonstrate the effect of task on group SSRT comparisons. Using only the estimates from the SR task would have lead us to conclude that there is insufficient evidence to support the hypothesis that SSRT differs between young and older adults, whereas there is a significant group difference in SSRT for the AR task, which we have demonstrated is less affected by skew and slowing. Thus, differences in go response strategies are not a trivial problem when comparing response inhibition efficiency between groups (e.g. patients, genetic variations, age) or conditions (e.g. proactive inhibition, complex stimuli).

In the AR task the response distribution is fairly normally distributed due to the fact that participants know when to respond, and there is naturally less slowing because of the inherent feedback about go performance. Therefore the AR task might provide a clearer picture of stopping performance. Note however that GoRTs become longer by $\sim 17 \mathrm{~ms}$ when introducing 
stop trials in the AR task, only to a lesser degree than in the CR paradigm where GoRTs are prolonged by $\sim 97 \mathrm{~ms}$. Similarly we have demonstrated before that GoRTs increase when the probability of a stop-signal increases (Coxon et al., 2006; Zandbelt \& Vink, 2010; Leunissen et al., 2016). Moreover, there was slightly more slowing in Experiment 2 than in Experiment 1. In Experiment 2 the target line did not change color according to go task performance, so there was no augmented feedback over and above seeing where the indicator stopped on each trial. Thus whilst the AR task stabilizes the response timing, it is not unaffected by proactive control.

A possible disadvantage of the AR task is that the inter-individual differences in SSRT are small (particularly in the young group). The restricted spread in SSRT values might hamper correlational approaches in small to medium sized samples. However, we, and others using the AR task, have been able to find evidence for correlations between SSRT and working memory function (Zandbelt et al., 2011), white matter microstructural properties (Coxon et al., 2012), age (Smittenaar et al., 2015), basal ganglia shape (Hermans et al., 2017) and effects of rTMS (Zandbelt et al., 2013a), suggesting the AR task does in fact have utility for research on interindividual differences. Nevertheless, task choice is a factor to take into consideration when the main goal is to focus on inter-individual differences. An alternative viewpoint is that SSRT is much less variable across individuals than we thought, and that correlations between SSRTs obtained with the CR task and other executive functions are due to a common element of choice/strategic behavior as opposed to a common inhibitory mechanism (Miyake et al., 2000). It is difficult to address this issue here due to the limited sample sizes and lack off additional measures alongside both versions of the stop-signal paradigm.

For young adults, we did find a significant positive relationship between SSRTs on the CR and AR paradigms $(r=0.48, p=0.025)$ and on the SR and AR paradigms $(r=0.69, p=0.005)$, meaning that participants who were fast and efficient in stopping their responses in the CR/SR paradigm were also fast and efficient at stopping in the AR paradigm. Suggesting that a common underlying neural mechanism might mediate response inhibition in all three tasks, which is consistent with the similarities in fMRI activations (Aron \& Poldrack, 2006; Li et al., 2008;

This article is protected by copyright. All rights reserved. 
Zandbelt \& Vink, 2010; Coxon et al., 2016; Leunissen et al., 2016). It also supports the idea that a single, general mechanism may underlie the ability to inhibit a wide variety of thoughts and actions (Logan \& Cowan, 1984; Logan, 1994; Aron, 2011). While significant, the shared variance between SSRTs on the CR/SR and AR task was modest (CR: 23\%, SR: 61\%). This suggests that there may also be differences in the underlying inhibitory mechanisms for inhibiting choice/simple reaction times and timed responses, or that inhibition takes place at different points in the motor hierarchy (Aron \& Poldrack, 2006). For example, if participants wait with initiating their motor response in the CR/SR paradigm then SSRT could reflect the cancelling of the development of a motor plan, instead of cancelling an already initiated response. We encourage researchers to address this outstanding question with larger samples and the latest developments in the model-based analysis of stop-signal data.

\section{Conclusions and Practical Guidelines}

In our opinion, the AR stop-signal task has the advantage of constraining go response behavior well enough to substantially reduce bias in SSRT related to the strategic emphasis on stop vs. go and the fundamental speed-accuracy tradeoff. We demonstrate that the SR task in particular, produces highly unreliable SSRT estimates. It has been suggested that bias due to skew of the response distribution can be minimized by adopting the integration method to estimate SSRT. Gradual slowing over the course of the experiment may also be reduced by providing clear instructions about the importance of responding as fast as possible and explaining the staircasing algorithm, and through reinforcement schedules tied to go task performance. Feedback on go task performance could be provided on a trial-by-trial (Verbruggen et al., 2004) or block (Mirabella et al., 2006) basis, and participants with significant slowing could be removed from the analysis. However, if strategic changes are of interest, such as when investigating proactive inhibition, providing feedback might not be possible. Moreover, one might need to remove a large proportion of the sample when excluding participants with

This article is protected by copyright. All rights reserved. 
significant slowing. Therefore, using a paradigm in which the presence of stop-signals does not drastically influence go task performance, such as the AR task, could provide a simple way to obtain more reliable measures of response inhibition performance when comparing different conditions or groups.

\section{Acknowledgements}

The authors thank René Clerckx for programming the tasks, and Johanna Mödden for assistance with data collection. This work was supported by the Interuniversity Attraction Poles program of the Belgian federal government [P7/11]. Additional support was provided by the Research Fund KU Leuven (OT/11/071) and Research Foundation - Flanders (FWO) (G.0483.10; G.0721.12). IL is funded by a FWO Post-Doctoral fellowship. ZP was funded by the European Commission through MOVE-AGE, an Erasmus Mundus Joint Doctorate Programme (2011-0015).

\section{Author Contributions}

J.P. developed the study concept. All authors contributed to the study design. Data collection was performed by I. Leunissen and Z. Potocanac. All authors contributed to the data analysis, interpretation and writing the manuscript. All authors approved the final version of the manuscript.

\section{Data accessibility}

The raw data used in this study has been submitted to a data repository. DOI: 10.6084/m9.figshare.4887053

\section{Conflict of interests}

No conflict of interest. 


\section{Abbreviations}

AR: anticipated response; CR: choice reaction; SR: simple reaction; SSRT: stop-signal reaction time; SSD: stop-signal delay; ITI: inter-trial interval; SD: standard deviation; RT: response time; ADHD: attention deficit hyperactivity disorder

\section{Figure Legends}

Figure 1. Visual displays. a) Anticipated response version of stop-signal paradigm. An indicator increased from the bottom up at constant velocity reaching the top in $1 \mathrm{~s}$. The indicator could be stopped by pressing/releasing the right mouse key. Posture for the different conditions is shown in the photographs below. Participants were required to stop the indicator as close to the target line (at $800 \mathrm{~ms}$ ) as possible. If the indicator stopped unexpectedly before the target, participants were instructed to withhold their response. b) Choice reaction version of stopsignal paradigm. A left or right oriented arrow was presented at the screen. Participants were instructed to press/release the corresponding mouse key as fast and as accurately as possible. Posture for the different conditions is shown in the photographs below. On infrequent occasions a square was presented shortly after the arrow, indicating that participants should withhold their response. c) Simple reaction version of the stop-signal paradigm. The go stimulus was a green square, which required pressing the button as fast as possible. On $30 \%$ of the trials the green square change into a red square, signaling that participants should try to prevent their response.

Figure 2. Independence of the stop and go process. a) Cumulative distributions of response latencies in non-successful stop trials (dashed lines) and go trials (full lines) for press (black) and release (grey) conditions. $\mathrm{AR}=$ anticipated response, $\mathrm{CR}=$ choice reaction, $\mathrm{SF}=$ stop fail. $\mathrm{b}$ ) Response times of failed stop trials increase with stop-signal delay for press (black) and release (grey) conditions. Error bars represent the standard deviation.

Figure 3. Go task performance. a) Upper panel: graphical presentation of explained variance in

This article is protected by copyright. All rights reserved. 
slowing of go response per subject and condition. Red crosses represent the mean. Lower panel: graphical presentation of tau estimation of ex-Gaussian fit of the go only trials and the go trials in the presence of stop-signals per condition. $A R p=A R$ press, $A R r=A R$ release,$C R p=C R$ press, $\mathrm{CRr}=\mathrm{CR}$ release b) Individual (grey) and group (black) ex-Gaussian probability density functions. Note that tau estimates were bounded below by zero, which could have resulted in a slight overestimation of tau in the AR task in the presence of a leftward skew.

Figure 4. Stop-signal reaction times. a) Relation between the stop-signal reaction time (SSRT) on the AR task and the CR task for each subject. b) Correlation between the SSRT of the press and release condition of the CR task, c) and the AR task. Dashed lines represent the 95\% confidence interval.

Figure 5. Independence of the stop and go process. a) Cumulative distributions of response latencies in non-successful stop trials (dashed lines) and go trials (full lines) for young (black) and older (grey) participants. $\mathrm{AR}=$ anticipated response, $\mathrm{SR}=$ simple reaction, $\mathrm{SF}=$ stop fail. $\mathrm{b}$ ) Response times of failed stop trials increase with stop-signal delay for young (black) and old (grey) participants. Error bars represent the standard deviation.

Figure 6. Go task performance. a) Upper panel: graphical presentation of explained variance in slowing of go response per subject and task. Red crosses represent the mean. Lower panel: graphical presentation of tau estimation of ex-Gaussian fit per group and task. b) Individual (grey) and group (black) ex-Gaussian probability density functions. Note that tau estimates were bounded below by zero, which could have resulted in a slight overestimation of tau in the AR task in the presence of a leftward skew.

Figure 7. Stop-signal reaction times. Relation between the stop-signal reaction time (SSRT) on the AR task and the SR task for young (a) and old participants (b). Dashed lines represent the $95 \%$ confidence interval.

This article is protected by copyright. All rights reserved. 


\section{References}

Aron, A.R. (2011) From Reactive to Proactive and Selective Control: Developing a Richer Model for Stopping Inappropriate Responses. Biological Psychiatry, 69, E55-E68.

Aron, A.R. \& Poldrack, R.A. (2006) Cortical and subcortical contributions to stop signal response inhibition: Role of the subthalamic nucleus. Journal of Neuroscience, 26, 2424-2433.

Band, G.P.H., van der Molen, M.W. \& Logan, G.D. (2003) Horse-race model simulations of the stop-signal procedure. Acta Psychologica, 112, 105-142.

Bissett, P.G. \& Logan, G.D. (2014) Selective stopping? Maybe not. Journal of Experimental Psychology: General, 143, 455-472.

Bland, J.M. \& Altman, D.G. (1996) Measurement error. BMJ, 313, 744.

Congdon, E., Mumford, J.A., Cohen, J.R., Galvan, A., Canli, T. \& Poldrack, R.A. (2012) Measurement and reliability of response inhibition. Front Psychol, 3, 37.

Coxon, J.P., Goble, D.J., Leunissen, I., Van Impe, A., Wenderoth, N. \& Swinnen, S.P. (2016) Functional Brain Activation Associated with Inhibitory Control Deficits in Older Adults. Cereb Cortex, 26, 12-22.

Coxon, J.P., Stinear, C.M. \& Byblow, W.D. (2006) Intracortical inhibition during volitional inhibition of prepared action. Journal of Neurophysiology, 95, 3371-3383.

Coxon, J.P., Stinear, C.M. \& Byblow, W.D. (2007) Selective inhibition of movement. Journal of Neurophysiology, 97, 2480-2489.

Coxon, J.P., Stinear, C.M. \& Byblow, W.D. (2009) Stop and Go: The Neural Basis of Selective Movement Prevention. Journal of Cognitive Neuroscience, 21, 1193-1203.

Coxon, J.P., Van Impe, A., Wenderoth, N. \& Swinnen, S.P. (2012) Aging and Inhibitory Control of Action: Cortico-Subthalamic Connection Strength Predicts Stopping Performance. Journal of Neuroscience, 32, 8401-8412.

Dunovan, K., Lynch, B., Molesworth, T. \& Verstynen, T. (2015) Competing basal ganglia pathways determine the difference between stopping and deciding not to go. Elife, 4, e08723.

Epstein, J.N., Langberg, J.M., Rosen, P.J., Graham, A., Narad, M.E., Antonini, T.N., Brinkman, W.B., Froehlich, T., Simon, J.O. \& Altaye, M. (2011) Evidence for higher reaction time variability for children with ADHD on a range of cognitive tasks including reward and event rate manipulations. Neuropsychology, 25, 427-441.

Forstmann, B.U., Tittgemeyer, M., Wagenmakers, E.J., Derrfuss, J., Imperati, D. \& Brown, S. (2011) The Speed-Accuracy Tradeoff in the Elderly Brain: A Structural Model-Based Approach. Journal of Neuroscience, 31, 17242-17249.

Greenhouse, I. \& Wessel, J.R. (2013) EEG signatures associated with stopping are sensitive to preparation. Psychophysiology, 50, 900-908.

This article is protected by copyright. All rights reserved. 
Heathcote, A., Brown, S. \& Mewhort, D.J. (2002) Quantile maximum likelihood estimation of response time distributions. Psychon Bull Rev, 9, 394-401.

Hermans, L., Beeckmans, K., Michiels, K., Lafosse, C., Sunaert, S., Coxon, J.P., Swinnen, S.P. \& Leunissen, I. (2017) Proactive Response Inhibition and Subcortical Gray Matter Integrity in Traumatic Brain Injury. Neurorehabil Neural Repair, 31, 228-239.

Lacouture, Y. \& Cousineau, D. (2008) How to use matlab to fit the ex-gaussian and other probability functions to a distribution of response times. Tutorials Quant Methods Psychol, 4, 35-45.

Lappin, J.S. \& Eriksen, C.W. (1966) Use of A Delayed Signal to Stop A Visual Reaction-Time Response. Journal of Experimental Psychology, 72, 805-811.

Leotti, L.A. \& Wager, T.D. (2010) Motivational influences on response inhibition measures. Journal of Experimental Psychology-Human Perception and Performance, 36, 430-447.

Leunissen, I., Coxon, J.P. \& Swinnen, S.P. (2016) A proactive task set influences how response inhibition is implemented in the basal ganglia. Hum Brain Mapp.

Li, C.S.R., Yan, P., Sinha, R. \& Lee, T.W. (2008) Subcortical processes of motor response inhibition during a stop signal task. Neuroimage, 41, 1352-1363.

Logan, G.D. (1994) On the ability to inhibit thought and action: A users' guide to the stop signal paradigm. In Dagenbach, D., Carr, T.H. (eds) Inhibitory processes in attention, memory and language. San Diego, CA, US: Academic Press, pp. 189-239.

Logan, G.D. \& Cowan, W.B. (1984) On the Ability to Inhibit Thought and Action - A Theory of An Act of Control. Psychological Review, 91, 295-327.

Logan, G.D., Cowan, W.B. \& Davis, K.A. (1984) On the ability to inhibit simple and choice reaction time responses: a model and a method. Journal of Experimental Psychology-Human Perception and Performance, 10, 276-291.

Logan, G.D., Schachar, R.J. \& Tannock, R. (1997) Impulsivity and inhibitory control. Psychological Science, 8, 60-64.

Matzke, D., Love, J. \& Heathcote, A. (2016) A Bayesian approach for estimating the probability of trigger failures in the stop-signal paradigm. Behav Res Methods.

Mirabella, G., Pani, P., Pare, M. \& Ferraina, S. (2006) Inhibitory control of reaching movements in humans. Experimental Brain Research, 174, 240-255.

Miyake, A., Friedman, N.P., Emerson, M.J., Witzki, A.H., Howerter, A. \& Wager, T.D. (2000) The unity and diversity of executive functions and their contributions to complex "frontal lobe" tasks: A latent variable analysis. Cognitive Psychology, 41, 49-100.

Oosterlaan, J., Logan, G.D. \& Sergeant, J.A. (1998) Response inhibition in AD/HD, CD, comorbid $\mathrm{AD} / \mathrm{HD}+\mathrm{CD}$, anxious, and control children: A meta-analysis of studies with the stop task. Journal of Child Neurology, 39, 411-425.

Pinheiro, J., Bates, D., DebRoy, S., Sarkar, D. \& Team, t.R.D.C. (2013) Nlme: Linear and Nonlinear Mixed Effects Models. . R package version.

This article is protected by copyright. All rights reserved. 
Ratcliff, R. \& Murdock, B.B. (1976) Retrieval Processes in Recognition Memory. Psychological Review, 83, 190-214.

Ratcliff, R., Thapar, A. \& McKoon, G. (2007) Application of the diffusion model to two-choice tasks for adults 75-90 years old. Psychol Aging, 22, 56-66.

Ridderinkhof, K.R., Band, G.P.H. \& Logan, G.D. (1999) A study of adaptive behavior: effects of age and irrelevant information on the ability to inhibit one's actions. Acta Psychologica, 101, 315-337.

Slater-Hammel, A.T. (1960) Reliability, Accuracy, and Refractoriness of a Transit Reaction. The Research Quarterly, 31, 217-228.

Smittenaar, P., Rutledge, R.B., Zeidman, P., Adams, R.A., Brown, H., Lewis, G. \& Dolan, R.J. (2015) Proactive and Reactive Response Inhibition across the Lifespan. PLoS One, 10, e0140383.

Szmalec, A., Demanet, J., Vandierendonck, A. \& Verbruggen, F. (2009) Investigating the role of conflict resolution in memory updating by means of the one-back choice RT task. Psychological Research-Psychologische Forschung, 73, 390-406.

Verbruggen, F., Chambers, C.D. \& Logan, G.D. (2013) Fictitious inhibitory differences: how skewness and slowing distort the estimation of stopping latencies. Psychological science, $24,352-362$.

Verbruggen, F., Liefooghe, B. \& Vandierendonck, A. (2004) The interaction between stop signal inhibition and distractor interference in the flanker and Stroop task. Acta Psychol (Amst), 116, 21-37.

Verbruggen, F. \& Logan, G.D. (2009) Models of response inhibition in the stop-signal and stopchange paradigms. Neurosci Biobeh Rev, 33, 647-661.

Verbruggen, F. \& Logan, G.D. (2015) Evidence for capacity sharing when stopping. Cognition, $142,81-95$.

Woodworth, R.S. \& Schlossberg, H. (1954) Experimental psychology. Holt, New York.

Zandbelt, B.B., Bloemendaal, M., Hoogendam, J.M., Kahn, R.S. \& Vink, M. (2013a) Transcranial Magnetic Stimulation and Functional MRI Reveal Cortical and Subcortical Interactions during Stop-signal Response Inhibition. Journal of Cognitive Neuroscience, 25, 157-174.

Zandbelt, B.B., Bloemendaal, M., Neggers, S.F.W., Kahn, R.S. \& Vink, M. (2013b) Expectations and violations: Delineating the neural network of proactive inhibitory control. Human Brain Mapping, 34, 2015-2024.

Zandbelt, B.B., van Buuren, M., Kahn, R.S. \& Vink, M. (2011) Reduced Proactive Inhibition in Schizophrenia Is Related to Corticostriatal Dysfunction and Poor Working Memory. Biological Psychiatry, 70, 1151-1158.

Zandbelt, B.B. \& Vink, M. (2010) On the Role of the Striatum in Response Inhibition. Plos One, 5.

Zou, G.Y. (2007) Toward using confidence intervals to compare correlations. Psychological Methods, 12, 399-413.

This article is protected by copyright. All rights reserved. 
Table 1. Performance Characteristics Experiment 1

\begin{tabular}{|c|c|c|c|c|c|c|c|c|}
\hline & & $\begin{array}{l}\text { AR press } \\
\text { Mean (sd) }\end{array}$ & $\begin{array}{l}\text { AR release } \\
\text { Mean (sd) }\end{array}$ & $\begin{array}{l}\text { CR press } \\
\text { Mean (sd) }\end{array}$ & $\begin{array}{l}\text { CR release } \\
\text { Mean (sd) }\end{array}$ & $\begin{array}{l}\text { Task } \\
\left(F, p \text {-value, } \eta^{2}\right)\end{array}$ & $\begin{array}{l}\text { Condition } \\
\left(F, p \text {-value, } \eta^{2}\right)\end{array}$ & $\begin{array}{l}\text { Task* } \\
\text { Condition } \\
\left(F, p \text {-value, } \eta^{2}\right)\end{array}$ \\
\hline \multirow[t]{4}{*}{ Go only } & Errors (\%) & $0.00(0.0)$ & $1.53(3.7)$ & $0.84(0.8)$ & $1.76(1.0)$ & & & \\
\hline & No response (\%) & $0.12(0.5)$ & $0.00(0.0)$ & $0.00(0.0)$ & $0.06(0.2)$ & & & \\
\hline & Mean GoRT (ms) & $-3.24(8.3)^{\S}$ & $-4.36(11.5)^{\S}$ & $363.16(37.5)$ & $352.39(31.5)$ & & & \\
\hline & SD GoRT (ms) & $29.81(6.4)$ & $32.54(6.0)$ & $59.34(14.4)$ & $57.97(17.5)$ & $54.51,<0.001,0.77$ & $0.241,0.630,0.02$ & $0.832,0.375,0.05$ \\
\hline \multirow[t]{10}{*}{ Stop signal } & Errors (\%) & $0.00(0.0)$ & $1.65(1.4)$ & $0.92(1.2)$ & $2.72(2.0)$ & & & \\
\hline & No response (\%) & $0.28(0.5)$ & $0.76(0.98)$ & $0.70(0.7)$ & $1.06(1.6)$ & & & \\
\hline & Mean GoRT (ms) & $11.66(6.6)^{\S}$ & $14.26(8.6)^{\S}$ & $461.98(65.8)$ & $446.51(77.6)$ & & & \\
\hline & SD GoRT (ms) & $31.45(5.4)$ & $35.31(7.4)$ & $93.27(24.7)$ & $85.65(27.8)$ & $97.40,<0.001,0.86$ & $0.55,0.469,0.03$ & $3.79,0.069,0.19$ \\
\hline & GoRT slowing over time ( $\mathrm{ms} /$ trial) & $-0.02(0.03)$ & $-0.01(0.05)$ & $0.06(0.3)$ & $0.08(0.2)$ & $8.00,0.005$ & $3.82,0.051$ & $0.06,0.807$ \\
\hline & Tau (ms) & $12.60(10.8)$ & $22.64(15.4)$ & $72.24(31.6)$ & $62.69(35.1)$ & $67.25,<0.001,0.81$ & $0.001,0.97,0.0$ & $2.70,0.120,0.14$ \\
\hline & Tau/sigma & $0.56(0.56)$ & $1.05(0.87)$ & $1.86(1.13)$ & $2.03(1.86)$ & $43.14,<0.001,0.73$ & $2.05,0.171,0.11$ & $4.23,0.056,0.209$ \\
\hline & Stop fail RT (ms) & $-5.48(8.5)^{\S}$ & $-6.55(9.5)^{\S}$ & $400.79(51.1)$ & $394.37(56.3)$ & & & \\
\hline & Probability of inhibition (\%) & $50.85(0.6)$ & $50.59(1.1)$ & $53.07(2.5)$ & $51.90(4.0)$ & $5.53,0.032,0.26$ & $2.79,0.115,0.15$ & $1.05,0.322,0.06$ \\
\hline & SSRT (ms) & $169.63(5.9)$ & $178.36(5.5)$ & $205.48(31.3)$ & $219.03(27.3)$ & $42.50,<0.001,0.73$ & $11.02,0.004,0.41$ & $0.48,0.498,0.03$ \\
\hline \multirow{3}{*}{$\begin{array}{l}\text { Reliability } \\
\text { SSRT }\end{array}$} & Ave absolute diff between split halves (ms) & $9.01(1.9)$ & $10.55(2.8)$ & $28.36(7.9)$ & $25.87(9.91)$ & $117.38,<0.001,0.88$ & $0.18,0.679,0.01$ & $3.16,0.095,0.17$ \\
\hline & $\sigma_{\mathrm{w}}(\mathrm{ms})$ & 8.08 & 9.49 & 25.90 & 24.08 & & & \\
\hline & Coefficient of repeatability (ms) & 22.39 & 26.27 & 71.75 & 66.70 & & & \\
\hline
\end{tabular}

$\mathrm{AR}=$ anticipated response, $\mathrm{CR}=$ choice reaction, $\mathrm{GoRT}=$ go response time, $\mathrm{SSRT}=$ stop signal reaction time, $\mathrm{SD}=$ standard deviation.

Statistics were not performed on the variables percent error and no response due to the high number of observations equal to zero, mean response times between the tasks were not

compared due to the different criteria for determining response time (i.e. relative to the target line for the AR task and relative to the imperative cue in the CR task).

$\S A R$ GoRT and stop fail RT are expressed relative to the target line (recorded response $-800 \mathrm{~ms}$ ).

This article is protected by copyright. All rights reserved. 
Table 2. Performance Characteristics Experiment 2

\begin{tabular}{|c|c|c|c|c|c|c|c|c|}
\hline & & Young & & Old & & Statistics & & \\
\hline & & $\begin{array}{l}A R \\
\text { Mean (sd) }\end{array}$ & $\begin{array}{l}S R \\
\text { Mean (sd) }\end{array}$ & $\begin{array}{l}A R \\
\text { Mean (sd) }\end{array}$ & $\begin{array}{l}S R \\
\text { Mean (sd) }\end{array}$ & $\begin{array}{l}\text { Task } \\
\text { (F, p-value) }\end{array}$ & $\begin{array}{l}\text { Group } \\
\text { (F,p-value) }\end{array}$ & $\begin{array}{l}\text { Task* } \\
\text { Group } \\
\text { (F, p-value) }\end{array}$ \\
\hline \multirow{10}{*}{ Stop signal } & Errors (\%) & $1.94(1.2)$ & $0.00(0.0)$ & $4.72(4.6)$ & $0.00(0.0)$ & & & \\
\hline & No response (\%) & $1.25(2.1)$ & $2.31(4.1)$ & $10.56(11.2)$ & $6.71(7.2)$ & & & \\
\hline & Mean GoRT (ms) & $39.19(23.9)^{\S}$ & $430.84(137.4)$ & $69.62(34.3)^{\S}$ & $580.01(149.5)$ & & & \\
\hline & SD GoRT (ms) & $40.99(7.0)$ & $126.13(50.7)$ & $59.89(15.6)$ & $144.74(27.2)$ & $128.0,<0.001,0.85$ & $3.90,0.061,0.14$ & $0.0,0.985,0.0$ \\
\hline & GoRT slowing over time (ms/trial) & $0.07(0.1)$ & $0.66(0.7)$ & $0.15(0.1)$ & $0.60(0.2)$ & $134.58,<0.001$ & $14.48,0.001$ & $6.61,0.01$ \\
\hline & Tau (ms) & $12.91(8.8)$ & $85.90(64.2)$ & $4.33(7.0)$ & $51.37(44.9)$ & $12.04,0.002,0.29$ & $1.511,0.217,0.20$ & $0.92,0.481,0.14$ \\
\hline & Tau/sigma & $0.39(0.28)$ & $0.10(0.18)$ & $2.62(3.96)$ & $0.56(0.53)$ & $5.58,0.027,0.20$ & $4.01,0.057,0.149$ & $2.40,0.135,0.09$ \\
\hline & Stop fail RT (ms) & $15.10(30.9)^{\S}$ & $364.65(124.4)$ & $27.12(44.6)^{\S}$ & $516.16(175.0)$ & & & \\
\hline & Probability of inhibition (\%) & $51.71(1.3)$ & $50.77(4.6)$ & $52.04(1.1)$ & $53.70(4.8)$ & $0.15,0.708,0.01$ & $2.80,0.108,0.11$ & $1.85,0.187,0.08$ \\
\hline & $\mathrm{SSRT}(\mathrm{ms})$ & $172.64(11.2)$ & $199.46(56.4)$ & $202.47(23.2)$ & $223.74(42.6)$ & $5.76,0.025,0.2$ & $5.74,0.025,0.2$ & $0.080 .785,0.0$ \\
\hline Reliability & Ave absolute diff between split halves (ms) & $12.45(3.2)$ & $119.51(53.6)$ & $19.86(6.4)$ & $68.43(22.9)$ & $85.19<0.001,0.79$ & $6.72,0.016,0.23$ & $12.04,0.002,0.34$ \\
\hline \multirow{2}{*}{ SSRT } & $\sigma_{\mathrm{w}}(\mathrm{ms})$ & 11.25 & 96.11 & 18.19 & 58.39 & & & \\
\hline & Coefficient of repeatability (ms) & 31.16 & 266.21 & 50.40 & 161.74 & & & \\
\hline
\end{tabular}

$\mathrm{AR}=$ anticipated response, $\mathrm{SR}=$ simple reaction, $\mathrm{GoRT}=$ go response time, $\mathrm{SSRT}=$ stop signal reaction time, $\mathrm{SD}=$ standard deviation.

Statistics were not performed on the variables percent error and no response due to the high number of observations equal to zero, mean response times between the tasks were not compared due to the different criteria for determining response time (i.e. relative to the target line for the $A R$ task and relative to the imperative cue in the SR task). ${ }^{\S} \mathrm{AR}$ GoRT and stop fail RT are expressed relative to the target line (recorded response $-800 \mathrm{~ms}$ ).

This article is protected by copyright. All rights reserved. 
A

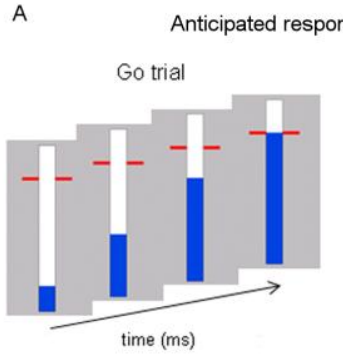

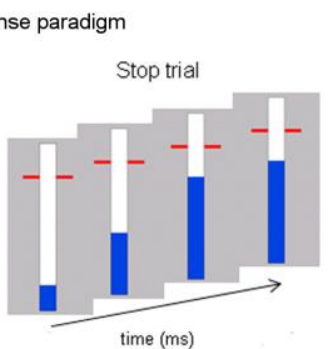

B

Choice reaction

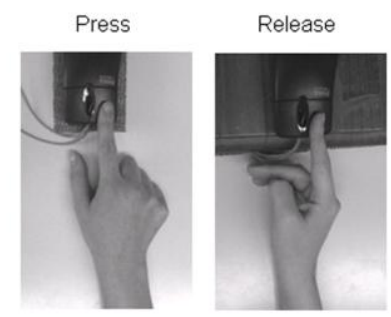

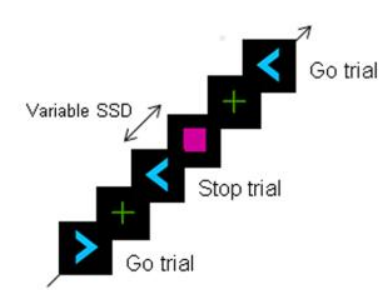

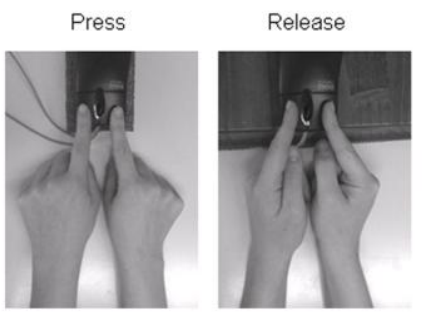

C

Simple reaction

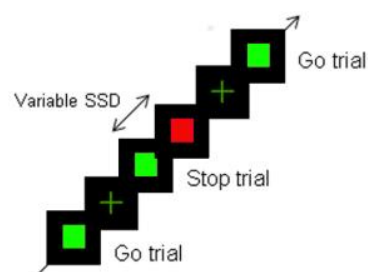

Press

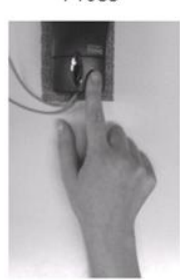

AR

CR

A
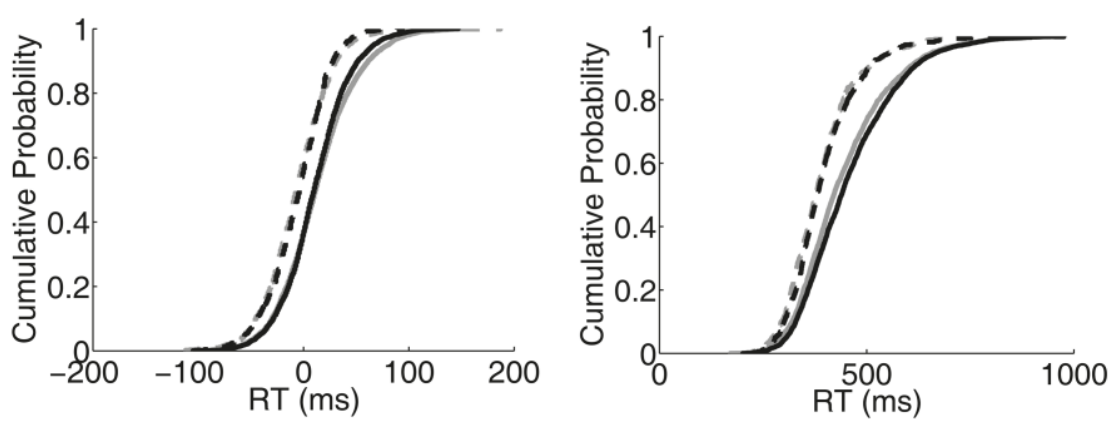

- Go press - - - SF press

Go release - - - SF release

B
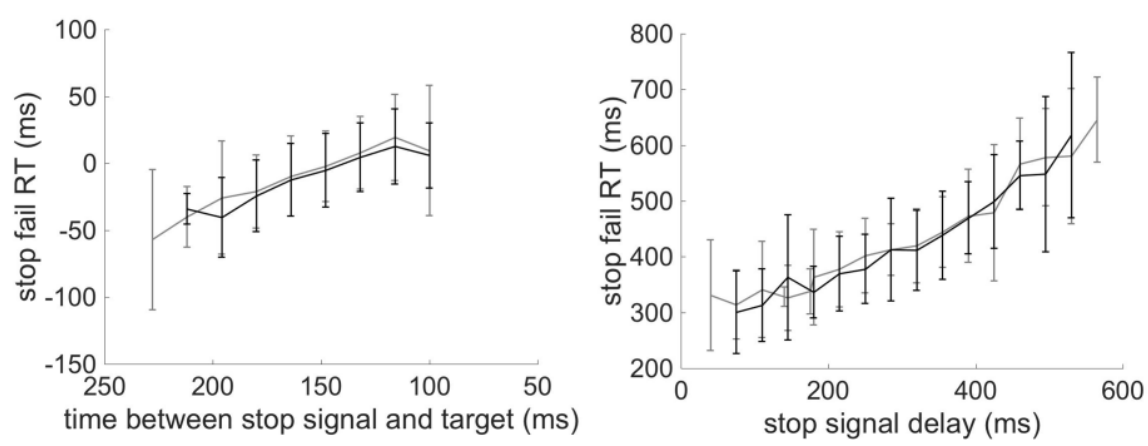

This article is protected by copyright. All rights reserved. 
A
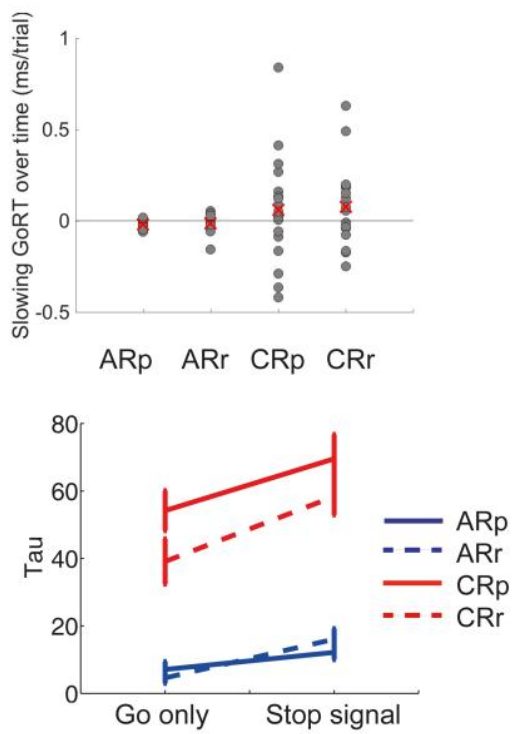

B

press
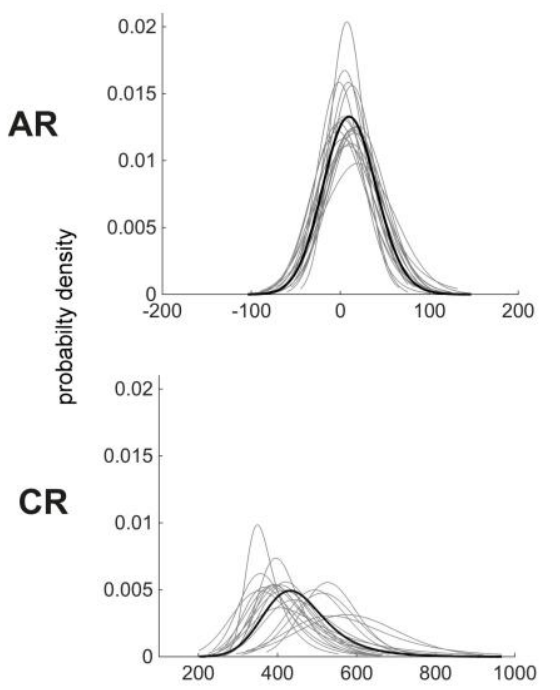

release
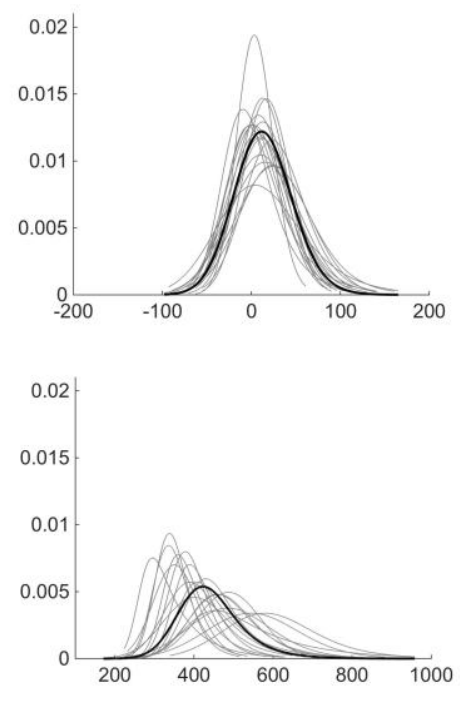

GoRT (ms)
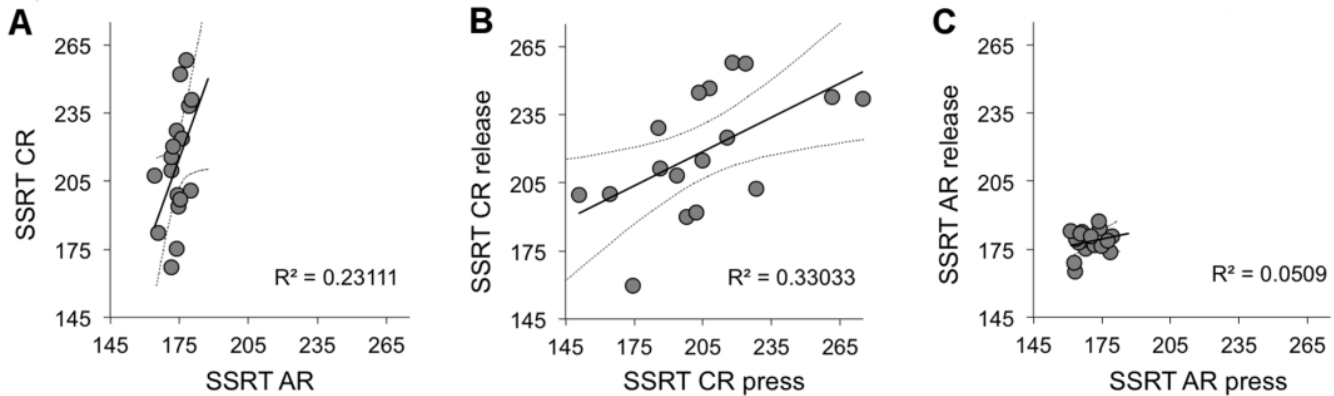

This article is protected by copyright. All rights reserved. 
AR
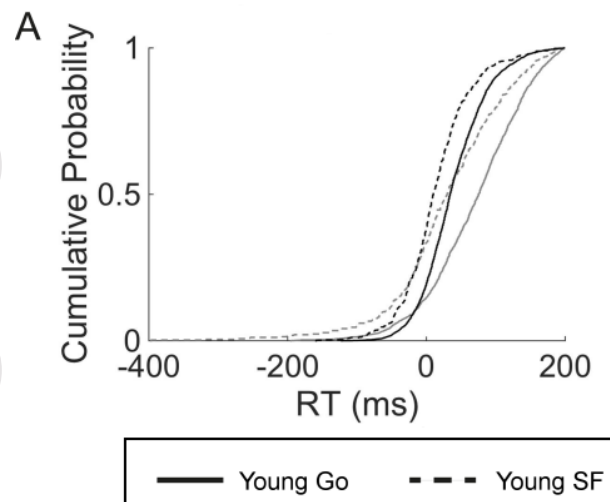

B

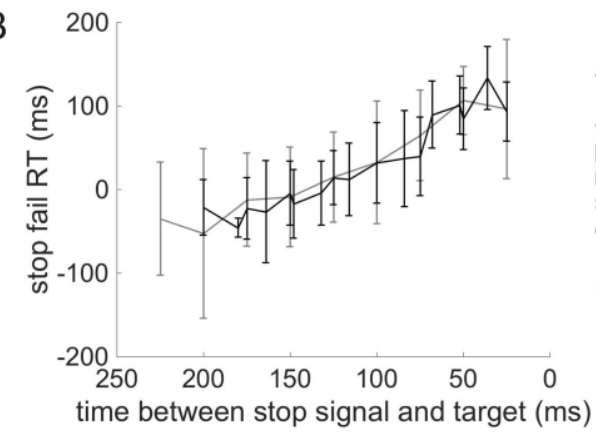

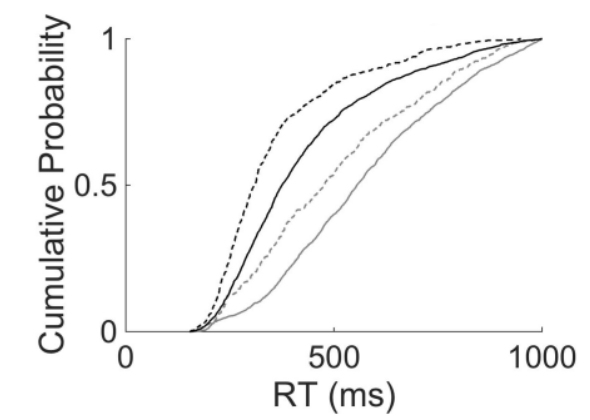

SR

Old Go

Old SF

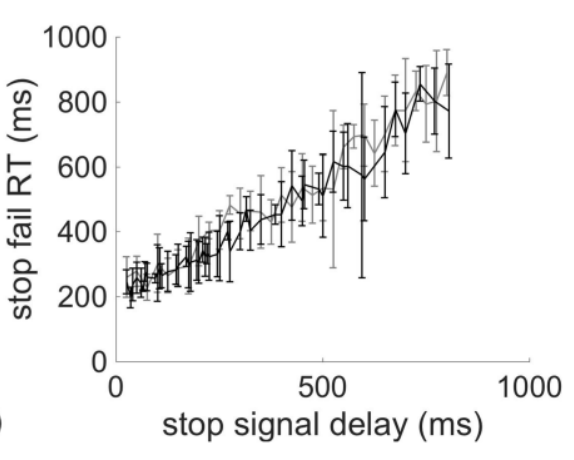

A
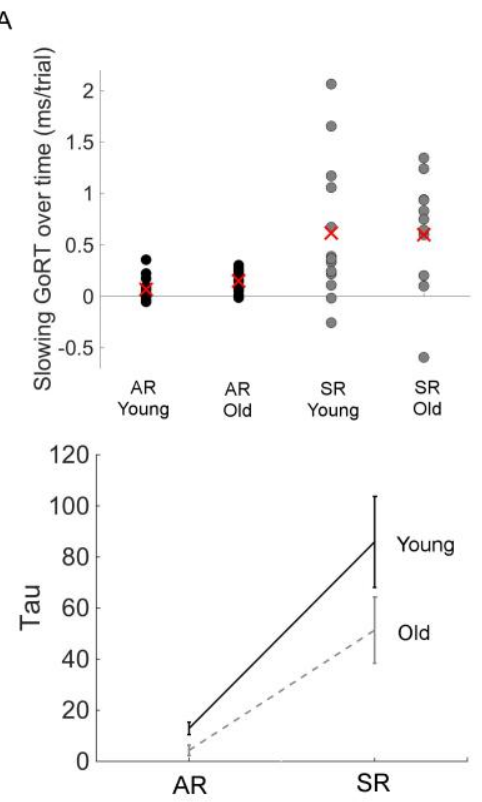

B
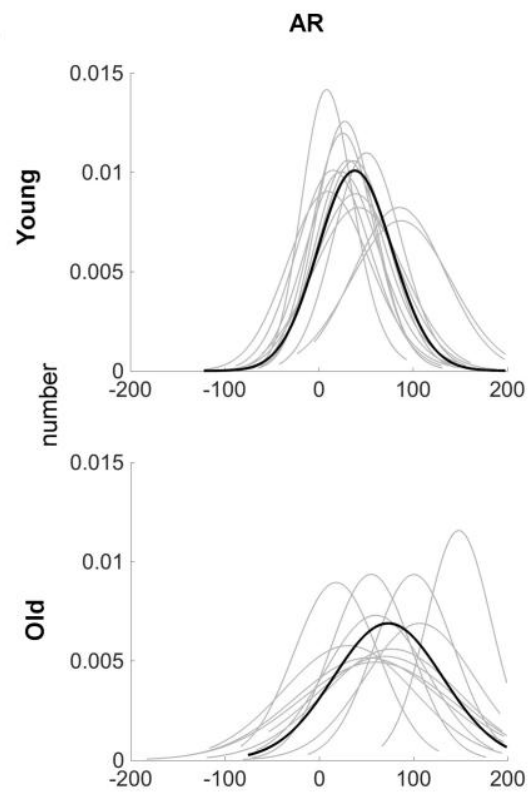

SR
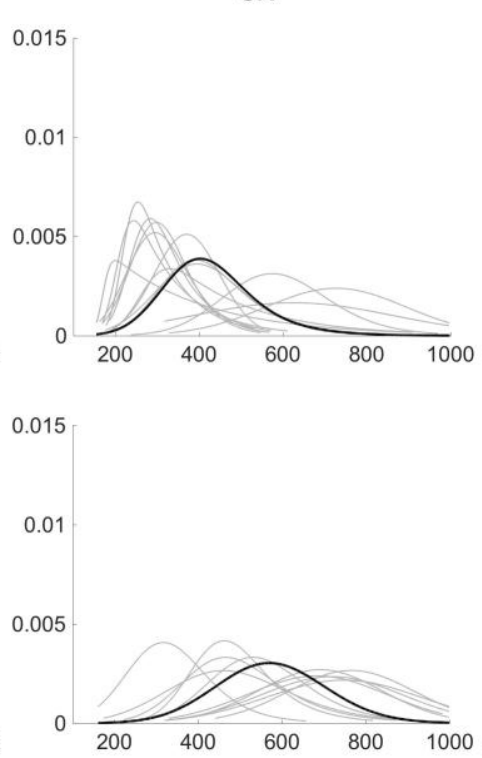

This article is protected by copyright. All rights reserved. 

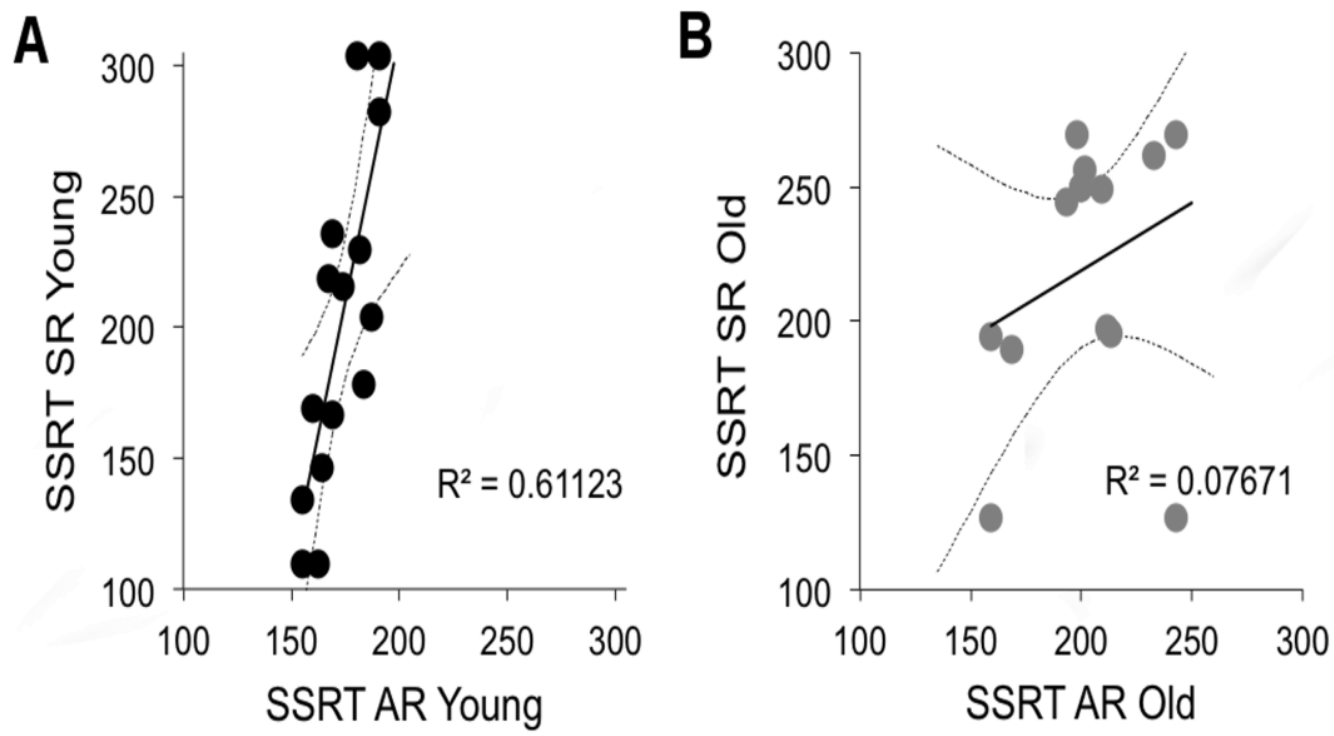

This article is protected by copyright. All rights reserved. 